\title{
Incomplete Information, Credibility and the Core*
}

\author{
Bhaskar Dutta $^{\dagger}$ \\ Rajiv Vohra
}

November 2003; Revised: March 2005.

\begin{abstract}
An appropriate (interim) notion of the core for an economy with incomplete information depends on the amount of information that coalitions can share. The coarse and fine core, as originally defined by Wilson (1978), correspond to two polar cases, involving no information sharing and arbitrary information sharing, respectively. We propose a new core notion, the credible core, which incorporates incentive compatibility constraints, and is based on the idea that a coalition can coordinate its potential objection to a status-quo over an event that can be credibly inferred from the nature of the objection being contemplated. We provide sufficient conditions ensuring non-emptiness of the credible core.
\end{abstract}

JEL Classification Nos. C71, D82, D51.

Keywords: Core, Asymmetric Information, Incentive Compatibility, Credibility.

*This is a substantial revision of an earlier paper with the same title. We thank two anonymous referees for helpful comments. Support from the ESRC Grant RES-000-220341(Dutta) and NSF Grant SES-0133113 (Vohra) is gratefully acknowledged.

$\dagger$ Department of Economics, University of Warwick, Coventry CV4 7AL, U.K.; b.dutta@warwick.ac.uk

$\ddagger$ Dean of the Faculty, Brown University, Providence, RI 02912-1857, U.S.A.; Rajiv_Vohra@brown.edu 


\section{Introduction}

Consider an exchange economy in which consumers have private information at the interim stage when state contingent contracts are made. Each agent knows her private information and has some probability assessment over the true information of others. We study an environment in which the only constraints on enforcing agreements are those arising from the incompleteness of information. A coalition can agree on a feasible state contingent contract (or net-trades) which is enforced by an agency using the private information reported by the agents. Naturally then, contracts need to be subjected to incentive compatibility constraints. An appropriate notion of the core provides a natural cooperative equilibrium concept for the problem of resource allocation in such an economy. One of the critical issues that arises in defining an appropriate core notion - and our central concern in the present paper - is the specification of the information that agents in a coalition are allowed to use in constructing an objection. In what way, if any, can members of a coalition share their private information? Put differently, over what kind of informational event is a coalition permitted to object? It should be borne in mind that this issue does not arise in defining the core at the ex ante stage; see Forges, Minelli and Vohra (2002) for additional discussion.

Wilson (1978), developed two distinct approaches that deal with this issue, and lead respectively to the notions of the coarse core and the fine core. ${ }^{1}$ The coarse core is based on the assumption that a coalition can focus its potential objection on an event if and only if the event is commonly known to all members of the coalition. Thus the act of forming an objecting coalition does not change the private information of any agent. The fine core is based on the idea that the act of forming a coalition allows all members of the coalition to decide how much of their private information they wish to share with each other.

Thus, the coarse and fine cores correspond to two extreme informational assumptions on coalitional behavior - the former rules out information sharing or leakage while the latter permits arbitrary sharing of information. We argue that both of these polar cases are subject to criticism. In particular, we show by means of an example that there may be circumstances in which it is reasonable for coalitions to coordinate their actions on an event which

\footnotetext{
${ }^{1}$ Wilson assumed that all information was publicly verifiable at the time of enforcement, and hence did not have to impose incentive compatibility. Our primary concern here will be with the incentive compatible versions of Wilson's core notions.
} 
is not a common knowledge event. Another example demonstrates that the fine core is also unreasonable since agents may not be able to pool their information in a credible manner.

In view of this discussion, it is natural to ask whether the theory can provide insights into the amount of private information that coalitions can be reasonably expected to pool. Recent work that considers such issues includes Forges (1994), Krasa (2000), Ichiishi and Sertel (1998), Lee and Volij (2002) and Volij (2000). In this paper our main aim is to make endogenous the information that is pooled in a coalition. We develop a notion of the core in which coalitions are allowed to coordinate their actions over an event that can be credibly inferred from the objection being contemplated.

Our notion of credible objections is meant to capture the following idea. Suppose a contract is under consideration as an objection by a coalition, and agent $i$ in the coalition claims that she is of type $s_{i}$. This claim is considered credible if agent $i$ would prefer the new contract to the status-quo if and only if she were indeed of type $s_{i}$. Other agents should, therefore, be able to infer $i$ 's statement regarding the informational event. This notion of credibility is closely related to that of credible updating used by Grossman and Perry (1986) in defining perfect sequential equilibrium; see also Cho and Kreps (1987) and Kahn and Mookherjee (1995).

The credible core is the set of allocations to which there is no credible objection. It lies between the incentive compatible fine core and the incentive compatible coarse core. We use several examples to illustrate the differences, and provide a positive result on the non-emptiness of the credible core in economies with quasi linear utilities. ${ }^{2}$ In particular, the credible core is nonempty in an auction of a single commodity in the case of private values or interdependent values satisfying the single crossing property.

\section{The Model}

In this Section we describe the basic model of an exchange economy with incomplete information. Since our main interest lies in analyzing an environment in which private information cannot be verified at the enforcement stage, we shall impose incentive compatibility constraints on all contracts.

\footnotetext{
${ }^{2}$ The non-emptiness issue is important because the incentive compatible coarse core (of which the credible core is a subset) can be empty in general, as shown in Vohra (1999) and Forges, Mertens and Vohra (2002).
} 
For this reason, we find it convenient to formulate private information in terms of agents' types.

Let $T_{i}$ denote the (finite) set of agent $i$ 's types. The interpretation is that $t_{i} \in T_{i}$ denotes the private information possessed by agent $i$. With $N=\{1, \ldots, n\}$ as the finite set of agents, let $T=\prod_{i \in N} T_{i}$. An information state for the economy refers to $t \in T$. We will use the notation $t_{-i}$ to denote $\left(t_{j}\right)_{j \neq i}$. Similarly $T_{-i}=\prod_{j \neq i} T_{j}$, and for any coalition $S$, a non-empty subset of $N, t_{S}=\left(t_{i}\right)_{i \in S}$ and $T_{S}=\prod_{i \in S} T_{i}$.

We assume that there is a (common) prior probability distribution $q$ defined on $T$. This is without loss of generality, except in section 5 , where we assume quasi linear preferences. ${ }^{3}$ Let $T^{*}=\{t \in T \mid q(t)>0\}$. We assume that none of the types is redundant in the sense that for every $i \in N$ and $t_{i} \in T_{i}$, there exists $t_{-i} \in T_{-i}$ such that $\left(t_{-i}, t_{i}\right) \in T^{*}$. We can now define, for $i \in N$ and $\bar{t}_{i} \in T_{i}$, the conditional probability of $t_{-i} \in T_{-i}$, given $\bar{t}_{i}$ as:

$$
q\left(t_{-i} \mid \bar{t}_{i}\right)=\frac{q\left(t_{-i}, \bar{t}_{i}\right)}{\sum_{t_{-i}^{\prime} \in T_{-i}} q\left(t_{-i}^{\prime}, \bar{t}_{i}\right)} .
$$

We assume that there are $l$ commodities, and each consumer has a consumption set $X_{i}=\mathbb{R}_{+}^{l}$ (for each state). Agent $i$ 's endowment is defined as a function $\omega: T_{i} \mapsto X_{i}$, where $\omega_{i}\left(t_{i}\right)$ denotes $i$ 's endowment when her type is $t_{i}$. Each consumer has a state dependent utility function $u_{i}: \mathbb{R}^{l} \times T \mapsto \mathbb{R}$. We will denote by $u_{i}(., t)$ the von Neumann-Morgenstern utility function of agent $i$ in state $t$. We assume that for each $i \in N$ and $t \in T, u_{i}(., t)$ is continuous and concave, and for all $x \in \mathbb{R}_{+}^{l}, u_{i}(x, t) \geq u_{i}(0, t)$.

A state contingent allocation is a function $x: T \mapsto \prod_{i} X_{i}$. Henceforth, we shall refer to a state contingent allocation simply as an allocation. The set of feasible allocations for the grand coalition is defined as:

$$
A_{N}=\left\{x: T \mapsto \prod_{i} X_{i} \mid \sum_{i \in N} x_{i}(t) \leq \sum_{i \in N} \omega_{i}\left(t_{i}\right), \text { for all } t \in T\right\} .
$$

An allocation for coalition $S$ is a function $x: T \mapsto \prod_{i \in S} X_{i}$, and is said to be feasible for coalition $S$ if

(a) $\sum_{i \in S} x_{i}(t) \leq \sum_{i \in S} \omega_{i}\left(t_{i}\right)$ for all $t \in T$.

\footnotetext{
${ }^{3}$ If no restrictions are imposed on preferences, then the common priors assumption can always be satisfied by appropriate rescaling of utilities and probabilities. However, quasilinearity imposes an additional restriction, and in that case the necessary rescaling may not be possible without violating quasi linearlity.
} 
(b) $x_{i}(t)=x_{i}\left(t^{\prime}\right)$ for all $i \in S, t, t^{\prime} \in T$ such that $t_{S}=t_{S}^{\prime}$.

Requirement (b) reflects the idea that a coalition cannot rely on the participation of outsiders in choosing its mechanism. If information becomes publicly verifiable at the enforcement stage there may be no reason to insist on (b). The set of feasible allocations for coalition $S$ is denoted $A_{S}$.

\subsection{The Coarse Core}

Suppose agents in a coalition do not (or cannot) share their private information. They can then coordinate their actions only over an event that is commonly known to them. A non-empty event $E \subseteq T$, is said to be a common knowledge event for coalition $S$ if $q\left(\hat{t}_{-i}, t_{i}\right)=0$ for all $i \in S, t \in E$ and $\left(\hat{t}_{-i}, t_{i}\right) \notin E$.

For an event $E \subseteq T$, define for each $i \in N$, the set of types of $i$ compatible with the event $E$ as

$$
E_{i}=\left\{t_{i} \in T_{i} \mid\left(t_{-i}^{\prime}, t_{i}\right) \in E \text { for some } t_{-i}^{\prime} \in T_{-i}\right\} .
$$

The conditional expected utility of consumer $i$ corresponding to allocation $x$, conditional on her being of type $t_{i}$, is

$$
U_{i}\left(x_{i} \mid t_{i}\right) \equiv \sum_{t_{-i}^{\prime} \in T_{-i}} q\left(t_{-i}^{\prime} \mid t_{i}\right) u_{i}\left(x_{i}\left(t_{-i}^{\prime}, t_{i}\right),\left(t_{-i}^{\prime}, t_{i}\right)\right) .
$$

Accordingly, $y \in A_{S}$ dominates $x \in A_{N}$ for $S$ over a common knowledge event $E$ if

$$
U_{i}\left(y_{i} \mid t_{i}\right)>U_{i}\left(x_{i} \mid t_{i}\right) \text { for all } t_{i} \in E_{i} \text { for all } i \in S .
$$

In addition to physical feasibility and domination, we also need to impose incentive compatibility constraints to ensure that an objecting allocation can be implemented by the coalition when private information cannot be verified.

Consider an allocation $y$. By pretending to be of type $s_{i}$, when her true type is $t_{i}$, agent $i$ can obtain the net-trade corresponding to the state $\left(t_{-i}, s_{i}\right)$ when the true state is $t$. Let the corresponding commodity bundle be denoted

$$
y_{i}\left(t_{-i}, s_{i} \mid t_{i}\right)=y_{i}\left(t_{-i}, s_{i}\right)-\omega_{i}\left(t_{-i}, s_{i}\right)+\omega_{i}\left(t_{i}\right) .
$$

This deception yields conditional expected utility

$$
U_{i}\left(y_{i}, s_{i} \mid t_{i}\right) \equiv \sum_{t_{-i}^{\prime} \in T_{-i}} q\left(t_{-i}^{\prime} \mid t_{i}\right) u_{i}\left(y_{i}\left(t_{-i}^{\prime}, s_{i} \mid t_{i}\right),\left(t_{-i}^{\prime}, t_{i}\right)\right) .
$$


We shall assume that a deception that leads to bankruptcy can never be profitable. This is equivalent to extending the domain of the utility function such that $u_{i}\left(y_{i}, t\right)=-\infty$ for all $y_{i} \notin \mathbb{R}_{+}^{l}$, for all $i \in N$ and $t \in T$.

An allocation $y$ is said to be incentive compatible for coalition $S$ if

$$
U_{i}\left(y_{i} \mid t_{i}\right) \geq U_{i}\left(y_{i}, s_{i} \mid t_{i}\right) \text { for all } s_{i}, t_{i} \in T_{i} \text {, for all } i \in S \text {. }
$$

Coalition $S$ has an incentive compatible, coarse objection to an incentive compatible allocation $x \in A_{N}$ if there exists $y \in A_{S}$ and an event $E$ that is common knowledge for $S$ such that $\left(D^{\prime}\right)$ and $\left(I C^{\prime}\right)$ hold. ${ }^{4}$ By the revelation principle, the set of incentive compatible allocations is identical to those which can be truthfully implemented as Bayesian Nash equilibria of a direct mechanism. An allocation can therefore be viewed as a mechanism. It is also worth pointing out that in this context, the assumption of free disposal which we implicitly made in defining feasible allocations for a coalition is no longer innocuous. A coalition may be able to do better if free disposal is allowed in the presence of incentive constraints; see Forges, Mertens and Vohra (2002) and Forges, Minelli and Vohra (2002) for examples.

The incentive compatible, coarse core consists of all incentive compatible allocations $x \in A_{N}$ to which there exists no incentive compatible, coarse objection. $^{5}$

\subsection{The Fine Core}

Suppose coalition $S$ considers an event $E \subseteq T$ over which to coordinate its actions through an allocation. The theory depends critically on the restrictions that are imposed on such an event. There are some basic restrictions which should always be imposed on such an event. Differences in various core notions will then depend on additional restrictions that might be imposed. It turns out that the basic restrictions we discuss below are already implicit

\footnotetext{
${ }^{4}$ One may argue that the incentive compatibility constraints as expressed in $\left(I C^{\prime}\right)$ are too strong; it should be enough to require these constraints over the common knowledge event E. Fortunately, as we will show in Proposition 2.1 below, this would not alter the notion of an incentive compatible, coarse objection.

${ }^{5}$ The corresponding core notion without incentive constraints is the coarse core of Wilson (1978). It consists of all allocations to which there exists no coarse objection (requiring (D') but not (IC')). Incentive constraints were incorporated into the coarse core in Vohra (1999). Allen (1992) was the first to introduce incentive constraints in studying the ex ante core.
} 
in an event which is commonly known to a coalition. ${ }^{6}$

To consider the possibility that a coalition may be able to act over an event that is not necessarily commonly known to all members of the coalition, suppose all members of coalition $S$ believe that the true state belongs to a non-empty set $E \subseteq T$. Clearly, there are some natural restrictions that ought to be imposed on $E$ (if $E$ is not a common knowledge event) for such beliefs to be reasonable. First, it must be the case that $E$ can be discerned without using the private information of those not in the coalition. So, if $S$ considers an event $E$ then a profile of types of agents outside $S$, $t_{-S}^{\prime} \in T_{-S}$, can be excluded from $E$ only if this is discernible with the private information of agents in $S$. Moreover, since all our domination notions will be based on evaluating conditional utilities, we can express this requirement as $E=E_{S} \times T_{-S}$. Second, $E$ must reflect independent claims by members of a coalition in a mechanism. In other words, $i$ 's claim that she is not of type $t_{i}$ cannot depend on claims by other members of $S$. So, $E_{S}$ must be the Cartesian product of the individual $E_{i}$ 's. Hence, $E=\left[\prod_{i \in S} E_{i}\right] \times T_{-S}$, where $E_{i} \subseteq T_{i}$ for all $i$. Finally, $E$ must be consistent with what each of the agents in the coalition know, given their private information. No agent, knowing her type, should rule out the possibility that the true state lies in $E$. In other words, for all $i \in S$ and $t_{i} \in E_{i}, q\left(E \mid t_{i}\right)>0$.

Thus, a non-empty event $E \subseteq T$ is said to be admissible for coalition $S$ if it is of the form:

$E=\prod_{i \in S} E_{i} \times T_{-S}$, where $E_{i} \subseteq T_{i}$ and $q\left(E \mid t_{i}\right)>0$ for all $t \in E$, for all $i \in S$.

Suppose a coalition can act over an admissible event $E$. The notion of domination used in $\left(D^{\prime}\right)$ needs to be modified to take account of the information that is contained in $E$.

The probability that agent $i$ assigns to $t \in E$, conditional on her type being $t_{i}$, and the belief that the true state is in $E$, is given by

$$
q\left(t_{-i} \mid t_{i}, E\right)=\frac{q\left(t_{-i} \mid t_{i}\right)}{q\left(E \mid t_{i}\right)}=\frac{q(t)}{\sum_{t_{-i}^{\prime} \in E_{-i}} q\left(t_{-i}^{\prime}, t_{i}\right)} .
$$

Note that if $E$ is an admissible event, this expression is well-defined since $q\left(E \mid t_{i}\right)>0$ for $t \in E$. We can now define for each $i \in S$ and a type $t_{i} \in E_{i}$,

\footnotetext{
${ }^{6}$ This will also make it clear that our definition of the incentive compatible, coarse core above is the same as the definition used in Vohra (1999).
} 
the conditional expected utility (conditional on $E$ ), for an allocation $x$ as

$$
U_{i}\left(x_{i} \mid t_{i}, E\right) \equiv \sum_{t_{-i}^{\prime} \in E_{-i}} q\left(t_{-i}^{\prime} \mid t_{i}, E\right) u_{i}\left(x_{i}\left(t_{-i}^{\prime}, t_{i}\right),\left(t_{-i}^{\prime}, t_{i}\right)\right)
$$

For coalition $S, y \in A_{S}$ dominates $x \in A_{N}$ over an admissible event $E$ if

$$
U_{i}\left(y_{i} \mid t_{i}, E\right)>U_{i}\left(x_{i} \mid t_{i}, E\right) \text { for all } t_{i} \in E_{i} \text { for all } i \in S .
$$

If coalition $S$ uses the information corresponding to an admissible event $E$, we shall need to consider incentive compatibility with respect to $E$. Given an allocation $y$ and an admissible event $E$, the conditional expected utility (conditional on the information provided by $E$ ) to agent $i$ of type $t_{i}$ by pretending to be of type $s_{i}$ is defined as

$$
U_{i}\left(y_{i}, s_{i} \mid t_{i}, E\right) \equiv \sum_{t_{-i}^{\prime} \in E_{-i}} q\left(t_{-i}^{\prime} \mid t_{i}, E\right) u_{i}\left(y_{i}\left(t_{-i}^{\prime}, s_{i} \mid t_{i}\right),\left(t_{-i}^{\prime}, t_{i}\right)\right) .
$$

An allocation $y$ is said to be incentive compatible over an admissible event $E$ for coalition $S$ if

$$
U_{i}\left(y_{i} \mid t_{i}, E\right) \geq U_{i}\left(y_{i}, s_{i} \mid t_{i}, E\right) \text { for all } s_{i}, t_{i} \in E_{i} \text {, for all } i \in S \text {. }
$$

Coalition $S$ has an incentive compatible, fine objection to an incentive compatible allocation $x \in A_{N}$ if there exists $y \in A_{S}$ and an admissible event $E$ for $S$ such that (D) and (IC) hold.

The incentive compatible, fine core consists of all incentive compatible allocations $x \in A_{N}$ to which there exists no incentive compatible, fine objection.

The fine core consists of all allocations $x$ to which there exists no fine objection, i.e., there exists no coalition $S$, an admissible event $E$ and a feasible allocation $y$ satisfying (D).

It is easy to see that an allocation $x$ that belongs to any of the cores we have defined must satisfy interim individually rationality in the sense that

$$
U_{i}\left(x_{i} \mid t_{i}\right) \geq U_{i}\left(\omega_{i} \mid t_{i}\right) \text { for all } i \in N, t_{i} \in T_{i}
$$

To clarify the essential difference between the incentive compatible, coarse core and the incentive compatible, fine core it is important to check that the domination and incentive compatibility conditions are, in fact, the same in 
each case. Conditions $(D)$ and $(I C)$ in defining a fine objection reflect the fact that agents update their prior probability assessments based on the pooled information. No such updating is required in a coarse objection since no additional information becomes available to any agent through the process of constructing an objection over a common knowledge event. It can also be shown that imposing admissibility on a common knowledge event would imply no loss of generality. Since both the coarse core and the fine core satisfy interim individual rationality, the essential difference between these core notions is explained in the following result.

Proposition 2.1 Suppose $x$ is interim individually rational. Coalition $S$ has an incentive compatible, coarse objection to $x$ (over an event $E$ which is common knowledge for $S$ ), if and only if $S$ has an incentive compatible, fine objection to $x$ over an (admissible) event $E^{\prime}$ which is common knowledge for $S$.

Proof: Suppose $y \in A_{S}$ is an incentive compatible, coarse objection of coalition $S$ to $x$ over a common knowledge event $E$. Let $E_{i}^{\prime}=E_{i}=\left\{t_{i} \in T_{i}\right.$ $\left(t_{-i}^{\prime}, t_{i}\right) \in E$ for some $\left.t_{-i}^{\prime} \in T_{-i}\right\}$, and let $E^{\prime}=\prod_{i \in S} E_{i}^{\prime} \times T_{-S}$. Of course, $E^{\prime}$ is a common knowledge event for $S$ since $E \subseteq E^{\prime}$. Since no type is redundant, and $E^{\prime}$ is a common knowledge event, it follows that $q\left(E^{\prime} \mid t_{i}\right)>0$ for all $t \in E^{\prime}$ and all $i \in S$. Thus $E^{\prime}$ is an admissible event. Since $E^{\prime}$ is a common knowledge event, it follows that for every $i \in S$ and $t \in E^{\prime}$, $q\left(t_{-i} \mid t_{i}, E^{\prime}\right)=q\left(t_{-i} \mid t_{i}\right)$. Thus, $U_{i}\left(y_{i} \mid t_{i}, E^{\prime}\right)=U_{i}\left(y_{i} \mid t_{i}\right)$. Since $\left(D^{\prime}\right)$ holds for all $t_{i} \in E_{i}=E_{i}^{\prime}$ for all $i \in S$, this implies (D). Of course, $\left(I C^{\prime}\right)$ implies $(I C)$ over $E^{\prime}$. Thus, $y$ is a fine objection by $S$ over a common knowledge (admissible) event $E^{\prime}$.

To prove the converse, suppose $y$ is an incentive compatible fine objection by $S$ over $E$, a common knowledge, admissible event $E$. Define $\tilde{y}$ such that for all $i \in S$,

$$
\tilde{y}_{i}(t)= \begin{cases}y_{i}(t) & \text { if } t \in E \\ \omega_{i}(t) & \text { otherwise }\end{cases}
$$

We now claim that $\tilde{y}$ is an incentive compatible coarse objection by $S$ to $x$ over the event $E$. Since $E$ is a common knowledge event, for all $t \in E$ and $i \in S$,

$$
U_{i}\left(\tilde{y}_{i} \mid t_{i}, E\right)=U_{i}\left(y_{i} \mid t_{i}, E\right)=U_{i}\left(y_{i} \mid t_{i}\right)
$$


Thus $(D)$ implies $\left(D^{\prime}\right)$. It remains to be shown that (IC) implies (IC'). Condition (IC) means that

$$
U_{i}\left(\tilde{y}_{i} \mid t_{i}\right) \geq U_{i}\left(\tilde{y}_{i}, s_{i} \mid t_{i}\right) \text { for all } s_{i}, t_{i} \in E_{i} \text { for all } i \in S .
$$

To establish (IC') we have to show that incentive compatibility holds even for $t_{i} \notin E_{i}$ or $s_{i} \notin E_{i}$. Suppose $t_{i} \notin E_{i}$. Now, the fact that $E$ is a common knowledge event implies that

$$
q\left(t_{-i}^{\prime}, t_{i}\right)>0 \text { only if } t_{-i}^{\prime} \notin E_{-i} .
$$

By construction,

$$
\tilde{y}_{i}\left(t_{-i}^{\prime}, s_{i}\right)=\omega_{i}\left(t_{i}\right) \text { for all } s_{i} \in T_{i} \text { and } t_{-i}^{\prime} \notin E_{-i} .
$$

Thus, if $t_{i} \notin E_{i}$, agent $i$ expects no-trade in every state that he believes possible:

$$
U_{i}\left(\tilde{y}_{i} \mid t_{i}\right)=U_{i}\left(\tilde{y}_{i}, s_{i} \mid t_{i}\right)=U_{i}\left(\omega_{i} \mid t_{i}\right) .
$$

The only remaining case is one in which $t_{i} \in E_{i}$ and $s_{i} \notin E_{i}$. This kind of lie results in no-trade, and cannot be profitable because

$$
U_{i}\left(y_{i} \mid t_{i}\right)>U_{i}\left(x_{i} \mid t_{i}\right) \geq U_{i}\left(\omega_{i} \mid t_{i}\right),
$$

where the first inequality follows from (D) and the second from the interim individual rationality of $x$.

Proposition 2.1 shows that we can take admissibility, $(D)$ and $(I C)$ to be the necessary conditions in defining an objection. The coarse core adds to these conditions the requirement that objections are only permitted over common knowledge events. Clearly then, the incentive compatible, fine core is a subset of the incentive compatible, coarse core.

It is important to keep in mind that both $(D)$ and $(I C)$ are defined with respect to an admissible event $E$, reflecting the updated probability assessments inherent in $E$. In particular, a fine objection by $S$ over a particular state, i.e., a fine objection by $S$ over an event $E=\prod_{i \in S}\left\{t_{i}\right\} \times T_{-S}$, makes condition $(I C)$ redundant. Thus an allocation in the incentive compatible fine core must necessarily be ex-post efficient in the sense of Holmström and Myerson (1983). ${ }^{7}$ Moreover, as Einy, Moreno and Shitovitz (2000) show, in

\footnotetext{
${ }^{7}$ The fact that an allocation in the fine core is ex-post efficient again points to the fact that an incentive compatible, fine objection may rely on an agent to believe unverifiable (and unreasonable) claims by another.
} 
an atomless economy, the fine core is a subset of the ex post core, i.e., a fine core allocation has the property that in each state, the allocation is a core allocation of the full information economy for that state.

As is well known, even in the two-consumer case, there might not exist any incentive compatible and interim individually rational allocation which is ex-post efficient (see, for example, Holmström and Myerson (1983) and Myerson and Satterthwaite (1983)). The incentive compatible, fine core may therefore be empty even in a two-consumer economy. ${ }^{8}$

The incentive compatible, coarse core is non-empty in (well-behaved) twoconsumer economies. It is also non-empty if preferences are linear (Ichiishi and Idzik (1996) and Vohra (1999)), if the information is non-exclusive (Vohra (1999)). ${ }^{9}$ However, there do exist well-behaved, three-consumer economies in which it is empty, as shown in Vohra (1999) and Forges, Mertens and Vohra (2002).

\section{Credible Information Pooling}

We shall argue that in some cases pooling of information is reasonable while in others it is not. Our aim is to formalize a notion of credible pooling of private information and a corresponding notion of a credible core. We begin with two simple, motivating examples. The first illustrates a situation in which information pooling seems reasonable, and provides a critique of the coarse core. The second illustrates a situation in which information pooling does not seem reasonable, and provides a critique of the fine core. These examples will also serve to introduce our notion of credibility.

\section{Example 3.1}

There are three consumers in an economy with two commodities. Each consumer $i$ can be of two possible types. Let $T_{i}=\left\{a_{i}, b_{i}\right\}$. Of the eight information states, only three arise with positive probability. These states are denoted

$$
t^{1}=\left(a_{1}, b_{2}, b_{3}\right), \quad t^{2}=\left(b_{1}, a_{2}, b_{3}\right) \quad t^{3}=\left(b_{1}, b_{2}, a_{3}\right) .
$$

\footnotetext{
${ }^{8}$ Wilson (1978) constructs a three-consumer example in which the fine core is empty. Recall that Wilson did not impose incentive compatibility.

${ }^{9}$ Forges (2004) shows that the incentive compatible coarse core is non-empty in twoperson assignment models when random mechanisms are allowed.
} 
All consumers have identical priors $q$, where $q(t)=1 / 3$ for $t \in T^{*}=\left\{t^{1}, t^{2}, t^{3}\right)$ and $q(t)=0$ for all $t \notin T^{*}$. In each state with positive probability there is exactly one consumer who is fully informed; consumer $i$ is the informed agent in state $t^{i}$.

The endowments are as follows:

$$
\omega_{i}\left(t_{i}\right)= \begin{cases}(1,0) & \text { if } t_{i}=b_{i} \\ (0.5,0.5) & \text { if } t_{i}=a_{i}\end{cases}
$$

For $x=\left(x_{1}, x_{2}\right) \in \mathbb{R}_{+}^{2}$, the state-dependent utility functions are as follows.

$$
\begin{aligned}
& u_{1}(x, t)= \begin{cases}1.5\left(x_{1}+x_{2}\right) & \text { if } t=t^{3} \\
x_{1}+x_{2} & \text { otherwise }\end{cases} \\
& u_{2}(x, t)= \begin{cases}1.5\left(x_{1}+x_{2}\right) & \text { if } t=t^{1} \\
x_{1}+x_{2} & \text { otherwise }\end{cases} \\
& u_{3}(x, t)= \begin{cases}1.5\left(x_{1}+x_{2}\right) & \text { if } t=t^{2} \\
x_{1}+x_{2} & \text { otherwise }\end{cases}
\end{aligned}
$$

Notice that both commodities are perfect substitutes. For an allocation $x$ let $\psi(x)$ denote the sum of the two commodities allocated to each consumer, i.e., $\psi_{i}(x, t)=x_{i 1}(t)+x_{i 2}(t)$. It can be shown that $x$ belongs to the incentive compatible coarse core if and only if

$\psi\left(x, t^{1}\right)=\left(1+\delta_{1}, 2-\delta_{1}, 0\right), \quad \psi\left(x, t^{2}\right)=\left(0,1+\delta_{2}, 2-\delta_{2}\right), \quad \psi\left(x, t^{3}\right)=\left(2-\delta_{3}, 0,1+\delta_{3}\right)$

where $\delta_{1}, \delta_{2}, \delta_{3} \in[0,1 / 3]$.

The incentive compatible, coarse core contains in particular the allocation $\bar{x}(t)$, where $\bar{x}_{i}(t)=\omega_{i}(t)$ for $t \notin T^{*}$ and

$$
\begin{aligned}
& \bar{x}\left(t^{1}\right)=((0.5,0.5),(2,0),(0,0)) \\
& \bar{x}\left(t^{2}\right)=((0,0),(0.5,0.5),(2,0)) \\
& \bar{x}\left(t^{3}\right)=((2,0),(0,0),(0.5,0.5))
\end{aligned}
$$

This allocation is not in the incentive compatible, fine core. ${ }^{10}$ Consumers 1 and 3 have a fine objection over the event $\left\{t^{1}\right\}$ since $\psi_{1}\left(\bar{x}, t^{1}\right)+\psi_{3}\left(\bar{x}, t^{1}\right)=1$,

\footnotetext{
${ }^{10}$ In fact, it can be shown, that in this example the fine core (with or without incentive constraints) is empty. The main difference between this example and Wilson's (1978) Example 2 is that in our example each agent's endowment depends on his own type.
} 
while their aggregate endowment of the two commodities is 2 . If private information can be shared, as is implicit in the notion of the fine core, then clearly $\bar{x}$ is not viable in state $t^{1}$. But, in the present example more can be said to justify a fine objection by agents 1 and 3 . Suppose the state is $t^{1}$, which consumer 1 knows. Consumer 3 knows that the true state is either $t^{1}$ or $t^{2}$. Consider an offer from consumer 1 to consumer 3 of the allocation $\tilde{x}(t)$, where

$$
\left(\tilde{x}_{1}(t), \tilde{x}_{3}(t)\right)= \begin{cases}((1.1,0),(0.4,0.5)) & \text { if } t=t^{1} \\ \left(\left(\omega_{1}(t), \omega_{3}(t)\right)\right. & \text { otherwise }\end{cases}
$$

In state $t^{1}$, the corresponding net-trades are $z_{1}\left(t^{1}\right)=(0.6,-0.5), z_{3}\left(t^{1}\right)=$ $(-0.6,0.5)$. In state $t^{1}$, the informed agent gives up 0.5 units of commodity 2 for 0.6 units of commodity 1 . Note that $t^{1}$ is the only state in which her endowments permit her to make this trade. While 3 does not know whether the true state is $t^{1}$ or $t^{2}$, she does know that the informed agent would be better off with this allocation only if the true state is $t^{1}$; if the state is actually $t^{2}$, the net-trade $(0.6,-0.5)$ is infeasible for agent 1 . The informed agent's claim, that the state is $t^{1}$, is credible and should, therefore, be accepted by agent 3. Acceptance of this allocation requires only that agent 3 infer (correctly) from the allocation that the state is $t^{1}$, not that 1 's private information becomes explicitly available to agent $3 .{ }^{11}$ In this respect this allocation offers a sensible objection to the status-quo. Agents should be able to coordinate on an event that can be inferred simply by the fact that all members of the coalition are willing to sign an allocation that is to their benefit only on the given event. In the present example, this makes it hard to justify the coarse core as the appropriate core notion.

We now give an example which shows that unlimited pooling of information, which is implicit in the definition of the fine core, may not be very appropriate under some circumstances.

\section{Example 3.2}

Consider a simpler version of Example 3.1 in which there is only one commodity, and each consumer has an endowment of 1 unit in each state. The information structure is the same as in the previous example.

\footnotetext{
${ }^{11}$ In the current example, the true state can be inferred from the allocation being proposed. However, this is not the case in general for our notion of a credible objection, to be defined in the next Section.
} 
The state-dependent utility functions are as follows.

$$
\begin{aligned}
& u_{1}(x, t)= \begin{cases}1.5 x & \text { if } t=t^{3} \\
x & \text { otherwise }\end{cases} \\
& u_{2}(x, t)= \begin{cases}1.5 x & \text { if } t=t^{1} \\
x & \text { otherwise }\end{cases} \\
& u_{3}(x, t)= \begin{cases}1.5 x & \text { if } t=t^{2} \\
x & \text { otherwise }\end{cases}
\end{aligned}
$$

It is easy to see that the incentive compatible, coarse core contains $\bar{x}(t)$, where $\bar{x}_{i}(t)=1$ for $t \notin T^{*}$, and

$$
\bar{x}\left(t^{1}\right)=(1,2,0), \quad \bar{x}\left(t^{2}\right)=(0,1,2), \quad \bar{x}\left(t^{3}\right)=(2,0,1) .
$$

This allocation is not in the incentive compatible, fine core. ${ }^{12}$ Consumers 1 and 3 have a fine objection over the event $\left\{t^{1}\right\}$ with an allocation $\tilde{x}\left(t^{1}\right)$ such that $\tilde{x}_{1}\left(t^{1}\right)=1+\epsilon$ and $\tilde{x}_{3}\left(t^{1}\right)=1-\epsilon$ for $\epsilon \in(0,1)$. In fact, every fine objection must be of this form. But agent 3 cannot infer from this allocation that consumer 1 is of type $a_{1}$ because consumer 1 would prefer the net trade $\epsilon$ in both states $t^{1}$ and $t^{2}$. Moreover, if the true state is $t^{2}$, consumer 3 by agreeing to the allocation $\tilde{x}$, and accepting 1 's claim that she is of type $a_{1}$, would be worse off compared to the status-quo $\bar{x}$. In this sense, the fine objection is not credible. The same argument holds for any fine objection to an allocation that belongs to the coarse core. In this example, therefore, the coarse core seems more reasonable than the fine core.

\section{The Credible Core}

The essential message from the previous examples is that the pooling of private information between members of a coalition should be permitted if and only if it can be justified as being credible. We now develop a notion of objections which incorporates this consideration.

Suppose each $i$ in coalition $S$ claims, independently, not to be of any type $\hat{t}_{i} \notin E_{i}$. This type, $\hat{t}_{i}$, cannot be ruled out by agent $j \in S$, with her private information, if

\footnotetext{
${ }^{12} \mathrm{As}$ in example 3.1, the incentive compatible, fine core is empty.
} 


$$
\text { for some } t \in E, \hat{t}_{i} \notin E_{i}, q\left(t_{-i}, \hat{t}_{i}\right)>0
$$

For each $i \in S$ let $V_{i}(E) \subseteq T_{i} \backslash E_{i}$ denote the set of all $\hat{t}_{i}$ satisfying (4.1). Of course, if the event $E$ is not a common knowledge event, $V_{i}(E) \neq \emptyset$ for some $i \in S$.

Our credibility criterion imposes the restriction that none of the types in $V_{i}(E)$ should select (or pretend) to be some type in $E_{i}$.

Given an admissible event $E$ for coalition $S$ define for each $i \in S$ and $\hat{t}_{i} \in V_{i}(E)$

$$
q\left(t_{-i} \mid \hat{t}_{i}, E\right)=\frac{q\left(t_{-i}, \hat{t}_{i}\right)}{\sum_{t_{-i}^{\prime} \in E_{-i}} q\left(t_{-i}^{\prime}, \hat{t}_{i}\right)} .
$$

Note that this expression is well-defined given the definition of $V_{i}(E)$.

For an event admissible for coalition $S$, we can now define for each $i \in S$ and a type $\hat{t}_{i} \in V_{i}(E)$, the conditional expected utility (conditional on $E$ ), of an allocation $x$ as

$$
U_{i}\left(x \mid \hat{t}_{i}, E\right)=\sum_{t_{-i}^{\prime} \in E_{-i}} q\left(t_{-i}^{\prime} \mid \hat{t}_{i}, E\right) u_{i}\left(x\left(t_{-i}^{\prime}, \hat{t}_{i}\right),\left(t_{-i}^{\prime}, \hat{t}_{i}\right)\right)
$$

Similarly, define the conditional expected utility of $x$ to $\hat{t}_{i} \in V_{i}(E)$ if $\hat{t}_{i}$ pretends to be of type $s_{i} \in T_{i}$ as

$$
U_{i}\left(x, s_{i} \mid \hat{t}_{i}, E\right) \equiv \sum_{t_{-i}^{\prime} \in E_{-i}} q\left(t_{-i}^{\prime} \mid \hat{t}_{i}, E\right) u_{i}\left(x_{i}\left(t_{-i}^{\prime}, s_{i} \mid \hat{t}_{i}\right),\left(t_{-i}^{\prime}, \hat{t}_{i}\right)\right)
$$

Note that this is well-defined since $\hat{t}_{i} \in V_{i}(E)$.

Suppose $x \in A_{N}, y \in A_{S}$ and $E$ is an admissible event for coalition $S$. An allocation $y$ is said to satisfy self-selection with respect to $x$ over $E$ if

$$
U_{i}\left(y, s_{i} \mid \hat{t}_{i}, E\right) \leq U_{i}\left(x \mid \hat{t}_{i}, E\right) \text { for all } \hat{t}_{i} \in V_{i}(E), s_{i} \in E_{i} \text { for all } i \in S \quad(\mathrm{SS}) \text {. }
$$

This constraint can be seen as an extension of (IC) to those types who are not supposed to be part of the objecting coalition. Notice that, as in (D) and (IC), the probabilities used in computing conditional expected utility, are those corresponding to the event $E$ over which the objection is supposed to take place. By the argument used in proving proposition 2.1, it can be shown that condition (SS) is equivalent to one in which this inequality is required to hold for all $s_{i} \in T_{i}$, not just all $s_{i} \in E_{i}$. 
Coalition $S$ is said to have a credible objection to an incentive compatible allocation $x \in A_{N}$ if there exists $y \in A_{S}$ and an admissible event $E$ such that (D), (IC) and (SS) are satisfied.

The credible core consists of all incentive compatible allocations to which there does not exist a credible objection.

We use Example 3.1 again to illustrate the nature of condition (SS). Consider again the allocation $\bar{x}(t)$ which we pointed out was not in the incentive compatible fine core. Let $S=\{1,3\}$. Then, the event $\left\{t^{1}\right\}$ is admissible for $S$ since 1 can discern that $t^{1}$ is the true state of the world. Consider the allocation $\tilde{x}$ specified there. Since 1 is the only informed agent, we need only check that (SS) is satisfied for 1 . Note that $V_{1}\left(t^{1}\right)=\left\{b_{1}\right\}$. As we have pointed out earlier, the net trade involved in $\tilde{x}$ is not feasible for 1 when her type is $b_{1}$. Hence, $\tilde{x}$ would give her a utility of $-\infty$ if she is of type $b_{1}$, but claims to be of type $a_{1}$. This shows that (SS) is satisfied, and so $\bar{x}$ is not in the credible core.

Remark 1. The credible core contains the incentive compatible, fine core, and is contained in the incentive compatible, coarse core. The first inclusion follows from the observation that an incentive compatible, fine objection is not required to satisfy (SS). To see the second inclusion, notice that if $E$ is a common knowledge event for $S$ then $V_{i}(E)=\emptyset$ for all $i \in S$, and (SS) is, therefore, vacuously satisfied. In example 3.1 , the credible core coincides with the incentive compatible, fine core, and in example 3.2 it coincides with the incentive compatible, coarse core. In the next section we will present an example in which these inclusions are strict, and all three cores are nonempty.

Remark 2. If incentive constraints, (IC), were to be dropped from the conditions defining the credible core, it would become identical to the fine core. This is so because a fine objection $y$ over $E$ by coalition $S$ is then equivalent to one in which agent $i$ is assigned 0 in every state $t$ such that $t_{-i} \in E_{-i}$ and $t_{i} \in V_{i}(E) .{ }^{13}$ Indeed, if types are verifiable as in Wilson (1978), then the fine core becomes a more appealing concept - there is no reason why members of a blocking coalition cannot share all their information since false communication will be detected.

\footnotetext{
${ }^{13}$ This observation does not apply to a notion of credibility in which instead of requiring that the wrong types lose we require that the other (uninformed) agents gain regardless. This is the idea used by Lee and Volij (2002) in defining the coarse + core, without imposing incentive constraints.
} 
The basic logic underlying our notion of the credible core is related to similar ideas used in other contexts. Most notably, it is similar to the concept of credible updating used by Grossman and Perry in defining a perfect sequential equilibrium. See also the discussion of the intuitive criterion in Cho and Kreps (1987) and the discussion in Kahn and Mookherjee (1995) regarding coalition proof Nash equilibrium under incomplete information. ${ }^{14}$ It is also related in spirit to the notion of durability studied by Holmström and Myerson (1983).

\section{Non-Emptiness of the Credible Core}

Recall that in example 3.1 the credible core is empty. Since utility functions in that example are linear, it follows that appealing to random allocations is not enough to establish non-emptiness. The aim of this section is to identify some sufficient conditions under which the credible core is non-empty. To do so, we shall assume throughout this section that utility functions are quasilinear. More precisely, we assume that the $l$-th commodity is money. The consumption set is then $X_{i}=\mathbb{R}_{+}^{l-1} \times \mathbb{R}$ and a typical element of $X_{i}$ is written as $x=(w, m)$. The utility function in each state is of the form:

$$
u_{i}((w, m), t)=v_{i}(w, t)+m .
$$

Note that the utility functions in example 3.1 are not quasi-linear, even though indifference curves are linear. ${ }^{15}$

We begin with an observation that relates to a special but important case. Suppose there are two agents, one of whom is uniformed and the other is informed. Consider a status-quo, $x$. If there is a credible objection, $y$, by the two agents over an event $E$, the self-selection constraints imply that an allocation that prescribes $y$ over $E$ and $x$ over $T \backslash E$ is also incentive compatible. Since this is also feasible in the two-consumer economy, it represents

\footnotetext{
${ }^{14} \mathrm{As}$ in the intuitive criterion, the speech by an agent making a claim about her type is: 'If I am of the wrong type I would not gain over the status-quo, so you should believe me'. Think of status-quo as the equilibrium. It can be broken if there is a way to signal information in a credible way which would make them all better off. In the coarse core, breaking the equilibrium is difficult because it has to be common knowledge that all types are better-off.

${ }^{15}$ That example is equivalent to one with quasi linear preferences only if one relaxes the assumption of a common prior.
} 
an incentive compatible coarse objection. ${ }^{16}$

Remark 3. In a quasi-linear economy with two agents, one informed and one uninformed, the credible core coincides with the incentive compatible, coarse core, and is, therefore, non-empty.

Coalition $S$ is said to have an ex post objection to $x \in A_{N}$ if there exists $y_{S} \in A_{S}$ and $t \in T$ such that $u_{i}\left(y_{i}, t\right)>u_{i}\left(x_{i}, t\right)$ for all $i \in S$. The ex post core is the set of all allocations in $A_{N}$ to which no coalition has an ex post objection. It is the set of allocations that correspond to the classical core of the (complete information) ex post economy $\mathcal{E}(t)=\left\{\left(X_{i}, u_{i}(., t), \omega_{i}(t)\right)\right\}$ for all $t \in T$.

Conditions under which the ex post core is non-empty are well known. In particular, it is non-empty in classical exchange economies with continuous, monotonic and convex preferences and convex consumption sets. Another case that will be of special interest to us is the one of an assignment model (Shapley and Shubik (1972)) in which Forges (2002) has established (allowing for random allocations) non-emptiness of the incentive compatible, coarse core. ${ }^{17}$ A leading example of such a model is that of several potential sellers and buyers, where each seller has one unit of an indivisible object, and preferences are quasi-linear. In this model, too, the ex post core is non-empty; see Shapley and Shubik (1972) and Quinzii (1984). In the quasi linear setting, this is useful for our purposes, as the following result shows.

Proposition 5.1 If utility functions are quasi-linear, every allocation in the ex post core belongs to the fine core, and the latter is, therefore, non-empty.

Proof. Suppose not, i.e, suppose there exists $x$ in the ex post core but not in the fine core. Then, there exists a coalition $S$, an admissible event $E$ and $y \in A_{S}$ such that for all $i \in S$, and all $t_{i} \in E_{i}$,

$\sum_{t_{-i}^{\prime} \in E_{-i}} q\left(t_{-i}^{\prime} \mid t_{i}, E\right) u_{i}\left(y_{i}\left(t_{-i}^{\prime}, t_{i}\right),\left(t_{-i}^{\prime}, t_{i}\right)\right)>\sum_{t_{-i}^{\prime} \in E_{-i}} q\left(t_{-i}^{\prime} \mid t_{i}, E\right) u_{i}\left(x_{i}\left(t_{-i}^{\prime}, t_{i}\right),\left(t_{-i}^{\prime}, t_{i}\right)\right)$.

Since

$$
q\left(t_{-i} \mid t_{i}, E\right)=\frac{q\left(t_{-i}, t_{i}\right)}{\sum_{t_{-i}^{\prime} \in E_{-i}} q\left(t_{-i}^{\prime}, t_{i}\right)}
$$

\footnotetext{
${ }^{16}$ Except for the fact that types not in $E$ are not strictly better-off. However, quasilinearity makes it possible to achieve this by transferring a small constant amount from the uninformed agent to the informed.

${ }^{17}$ Whether her result can be extended to cover the credible core remains an open question.
} 
we can re-write this as:

$\sum_{t_{-i}^{\prime} \in E_{-i}} q\left(t_{-i}^{\prime}, t_{i}\right) u_{i}\left(y_{i}\left(t_{-i}^{\prime}, t_{i}\right),\left(t_{-i}^{\prime}, t_{i}\right)\right)>\sum_{t_{-i}^{\prime} \in E_{-i}} q\left(t_{-i}^{\prime}, t_{i}\right) u_{i}\left(x_{i}\left(t_{-i}^{\prime}, t_{i}\right),\left(t_{-i}^{\prime}, t_{i}\right)\right)$.

Since this inequality holds for all $t_{i} \in E_{i}$ and $i \in S$, it follows that

$$
\sum_{t \in E} q(t) \sum_{i \in S} u_{i}\left(y_{i}(t), t\right)>\sum_{t \in E} q(t) \sum_{i \in S} u_{i}\left(x_{i}(t), t\right)
$$

Thus, there exists $t \in E$ such that $q(t)>0$ and

$$
\sum_{i \in S} u_{i}\left(y_{i}(t), t\right)>\sum_{i \in S} u_{i}\left(x_{i}(t), t\right) .
$$

Since utility functions are quasi-linear this means that coalition $S$ has an ex post objection in state $t$. But this contradicts the supposition that $x$ belongs to the ex post core.

Of course, this result does not address incentive issues, and for that reason alone does not directly help in terms of non-emptiness of the credible core. However, if we can find an ex post core allocation that is incentive compatible, then it would follow that such an allocation belongs to the credible core. We record this implication as a corollary.

Corollary 5.1 If utility functions are quasi-linear and there exists an incentive compatible allocation, $x$, in the ex post core, then $x$ belongs to the incentive compatible fine core, and, therefore, to the credible core.

In the general, the condition that there exist an incentive compatible allocation in the ex post core is a strong one. In fact, it is not to be expected even in simple buyer-seller models with two-sided incompleteness of information, as shown by Myerson and Satterthwaite (1983). However, a special case in which this property is known to hold is one in which the values of one side of the market are known. For example if the sellers' values are know, a buyer optimal mechanism in the ex post core is incentive compatible; see for example section 8.4 in Roth and Sotomayor (1990). This yields the following.

Corollary 5.2 In the assignment model, the credible core is non-empty if the valuations of one side of the market are known and values are private. 
Of course, the case of a single seller is a special case of the assignment model, and the second-price auction yields an allocation in the credible core. It is possible to generalize this positive result, for a single unit auction, in another direction. Suppose buyers have interdependent values. Even in this case, it is easy to see that the ex post core consists, in each state, of allocating the object to a highest valuation agent, and transferring from this agent to the seller an amount of money between the highest and the second highest valuation. If valuations satisfy the single crossing property, the generalized Vickrey-Clarke-Groves mechanism yields such an allocation as an ex post equilibrium, which implies incentive compatibility; see Proposition 10.1 in Krishna (2002). This gives us our next result.

Corollary 5.3 The credible core is non-empty in an auction model where valuations satisfy the single crossing property.

Another case in which non-emptiness can be established is one in which information is non-exclusive. Postlewaite and Schmeidler(1986) use this terminology to describe an information structure in which the information of any individual agent can be deduced by pooling the information of all the others. Formally, information is non-exclusive if for every $i \in N$ and $t \in T^{*}$, $q\left(t_{i} \mid t_{-i}\right)=1$.

When information is non-exclusive, any unilateral deception can be detected, and so incentive compatibility is easy to satisfy. In particular, for any interim individually rational allocation $x \in A_{N}$, there is another allocation $\hat{x} \in A_{N}$ which gives every $i$ the same interim utility as $x$, and is incentive compatible. ${ }^{18}$ Hence,

Corollary 5.4 If information is non-exclusive and the ex post core is nonempty, then the credible core is non-empty.

While corollaries 5.2 and 5.3 refer to specific allocations, the credible core is generally larger, as we will now illustrate. The following example will also serve to show that the incentive compatible fine core can be strictly contained in the credible core, which in turn can be a strict subset of the incentive compatible, coarse core.

\section{Example 5.1}

\footnotetext{
${ }^{18}$ See Lemma 3.1 in Vohra(1999).
} 
Suppose there are 2 buyers with the following valuations:

$$
\left(t_{1}^{H}, t_{1}^{L}\right)=(10,5), \quad\left(t_{2}^{H}, t_{2}^{L}\right)=(6,1) .
$$

The seller has 0 as reservation value. Types are equally likely.

Ex-post efficient allocations are characterized simply by the property that the object be allocated to buyer 2 in state $\left(t_{1}^{L}, t_{2}^{H}\right)$ and to buyer 1 in all other states. The allocation corresponding to the second price auction is one such allocation that has the stronger property of belonging to the ex post core. Since this allocation is also incentive compatible, it follows from Corollary 5.1 that it belongs to the incentive compatible fine core. The net utilities of buyer 1 , buyer 2 , and the seller, associated with this allocation are shown in the following table.

\begin{tabular}{c|c|c|}
\multicolumn{1}{c}{$t_{2}^{H}$} & \multicolumn{1}{c}{$t_{2}^{L}$} \\
\cline { 2 - 3 }$t_{1}^{H}$ & $(4,0,6)$ & $(9,0,1)$ \\
\cline { 2 - 3 }$t_{1}^{L}$ & $(0,1,5)$ & $(4,0,1)$ \\
\cline { 2 - 3 } & &
\end{tabular}

For instance, in state $\left(t_{1}^{H}, t_{2}^{H}\right)$ buyer 1 receives the object and pays the seller 6. As pointed out above, this allocation belongs to the incentive compatible fine core, and therefore also to the credible core and the incentive compatible coarse core. The fact that all these cores are non-empty in this setting makes it possible to make a meaningful comparison between the various cores, as summarized by the following observations.

The incentive compatible fine core contains allocations that are not in the ex-post core.

In a model with asymmetric information, it is possible to sustain some kind of core stability while providing an 'informational rent'; see, for instance, Example 3.1 in Vohra (1999). In the present example, consider the following modification to the second-price auction mechanism in the states where buyer 1 is of the low type:

\begin{tabular}{c|c|c|}
\multicolumn{1}{c}{} & \multicolumn{1}{c}{$t_{2}^{H}$} & \multicolumn{1}{c}{$t_{2}^{L}$} \\
\cline { 2 - 3 }$t_{1}^{H}$ & $(4,0,6)$ & $(9,0,1)$ \\
\cline { 2 - 3 }$t_{1}^{L}$ & $(0,0.5,5.5)$ & $(4,0.5,0.5)$ \\
\cline { 2 - 3 } & &
\end{tabular}

This is not in the ex-post core because in state $\left(t_{1}^{L}, t_{2}^{L}\right)$, buyer 2 receives a transfer even though the commodity is transferred to buyer 1. However, this allocation is in the fine core. Even if buyer 1 and the seller can share 
their private information, they wouldn't know the type of buyer 2. When buyer 1 is of the low type, the maximum expected surplus she can get with the seller is 5 . And that cannot provide an improvement over the statusquo. The transfer to buyer 2 in $\left(t_{1}^{L}, t_{2}^{L}\right)$ can be seen as a result of buyer 2 's contribution to the aggregate surplus in state $\left(t_{1}^{L}, t_{2}^{H}\right)$.

The credible core contains allocations that are not in the incentive compatible fine core.

Consider the allocation with the following utility profile.

$$
\begin{array}{c|c|c|}
\multicolumn{1}{c}{} & \multicolumn{1}{c}{t_{2}^{H}} & \multicolumn{1}{c}{t_{2}^{L}} \\
\cline { 2 - 3 } t_{1}^{H} & (4,1,5) & (6,0,4) \\
\cline { 2 - 3 } t_{1}^{L} & (0,1,5) & (0,0,5) \\
\cline { 2 - 3 } &
\end{array}
$$

The fact that buyer 2 receives a positive transfer in state $\left(t_{1}^{H}, t_{2}^{H}\right)$ means that this mechanism is not in the ex post core, and hence not in the fine core. However, this allocation does belong to the credible core. When agent 1 is of the high type, her expected payoff in this allocation is 5 . So, to make an objection, she has to offer a price less than 5, (but greater than 4.5). But, then buyer 1 of type $t_{1}^{L}$ would also make this offer.

The incentive compatible coarse core contains allocations that are not in the credible core.

The following allocation belongs to the incentive compatible coarse core.

\begin{tabular}{c|c|c|}
\multicolumn{1}{c}{} & \multicolumn{1}{c}{$t_{2}^{H}$} & \multicolumn{1}{c}{$t_{2}^{L}$} \\
\cline { 2 - 3 }$t_{1}^{H}$ & $(4,1,5)$ & $(5,0,5)$ \\
\cline { 2 - 3 }$t_{1}^{L}$ & $(0,0,6)$ & $(0,0,5)$ \\
\cline { 2 - 3 }
\end{tabular}

However, this is not in the credible core. Buyer 1 of the high type can signal his type in a credible way to avoid a transfer to buyer 2 in $\left(t_{1}^{H}, t_{2}^{H}\right)$. In particular, if buyer 1 offers to buy the object at a price of 5.1, it is obvious that she is of the high type (since the low type's valuation is 5), and both she and the seller are better-off with such an allocation. 


\section{References}

Allen, B., 1992. Incentives in Market Games with Asymmetric Information: the Core. CORE Discussion Paper 9221, Université Catholique de Louvain.

Cho, I-K., Kreps, D., 1987. Signaling Games and Stable Equilibria. Quarterly Journal of Economics 102, 179-222.

Einy, E., Moreno, D., Shitovitz, B., 2000. On the Core of an Economy with Differential Information. Journal of Economic Theory 94, 262-270.

Forges, F., 1994. A Note on Pareto Optimality in Differential Information Economies. Economics Letters 46, 27-31.

Forges, F., 2004. The Ex Ante Incentive Compatible Core of the Assignment Game. Mathematical Social Sciences 47, 135-151.

Forges, F., Mertens, J-F., Vohra, R., 2002. The Ex Ante Incentive Compatible Core in the Absence of Wealth Effects. Econometrica 70, 1865-1892.

Forges, F., Minelli, E., Vohra, R., 2002. Incentives and the Core of an Exchange Economy: A Survey. Journal of Mathematical Economics 38, $1-41$.

Grossman, S.J., Perry, M., 1986. Perfect Sequential Equilibrium. Journal of Economic Theory 39, 97-119.

Holmström, B., Myerson, R., 1983. Efficient and Durable Decision Rules with Incomplete Information. Econometrica 51, 1799-1819.

Ichiishi, T., Idzik, A., 1996. Bayesian Cooperative Choice of Strategies. International Journal of Game Theory 25, 455-473.

Ichiishi, T., Sertel, M., 1998. Cooperative Interim Contract and Re-Contract: Chandler's M-Form firm. Economic Theory 11, 523-543.

Kahn, C., Mookherjee, D., 1995. Coalition Proof Equilibrium in an Adverse Selection Insurance Economy. Journal of Economic Theory 66, 113-138.

Krasa, S., 2000. Unimprovable Allocations in Economies with Incomplete Information. Journal of Economic Theory 87, 144-168.

Krishna, V., 2002. Auction Theory, Academic Press,.

Lee, D., Volij, O., 2002. The Core of Economies with Asymmetric Information: An Axiomatic Approach. Journal of Mathematical Economics 38, 43-63.

Myerson, R., Satterthwaite, M., 1983. Efficient mechanisms for bilateral trading. Journal of Economic Theory 23, 265-281.

Postlewaite, A., Schmeidler, D., 1986. Implementation in Differential Information Economies. Journal of Economic Theory 33, 14-33. 
Quinzii, M., 1984. Core and Competitive Equilibria with Indivisibilities. International Journal of Game Theory 13, 41-60.

Roth, A., Sotomayor, M., 1990. Two-Sided Matching, a study in gametheoretic modeling and analysis, Cambridge University Press, Cambridge.

Shapley, L., Shubik, M., 1972. The Assignment Game I: the Core. International Journal of Game Theory 1, 111-130.

Vohra, R., 1999. Incomplete Information, Incentive Compatibility and the Core. Journal of Economic Theory 86, 123-147.

Volij, O., 2000. Communication, Credible Improvements and the Core of an Economy with Asymmetric Information. International Journal of Game Theory 29, 63-79.

Wilson, R., 1978. Information, Efficiency and the Core of an Economy. Econometrica 46, 807-816. 